\title{
Activation of AMP-activated protein kinase reduces collagen production via p38 MAPK in cardiac fibroblasts induced by coxsackievirus B3
}

\author{
SHENGYANG JIANG ${ }^{1}$, DONGLIN JIANG ${ }^{2}$, PENG ZHAO ${ }^{2}$, XINLONG HE ${ }^{2}$, \\ SHUNLI TIAN $^{3}$, XUEMING WU $^{1}$ and YIJIA TAO ${ }^{1}$
}

${ }^{1}$ Department of Cardiology; ${ }^{2}$ Clinical Central Laboratory, The Third Affiliated Hospital of Nantong University, Wuxi Institute of Integrated Traditional Chinese and Western Medicine, Wuxi, Jiangsu 214041; ${ }^{3}$ Department of Geratology,

Tianjin Geriatric Institute, Tianjin Medical University General Hospital, Tianjin 300052, P.R. China

Received June 27, 2015; Accepted April 26, 2016

DOI: $10.3892 / \mathrm{mmr} .2016 .5319$

\begin{abstract}
Collagen deposition is the major cause of myocardial fibrosis, contributing to impaired cardiac contractile function in coxsackie virus B3 (CVB3)-infected hearts. Adenosine monophosphate-activated protein kinase (AMPK) has been considered as a cellular fuel gauge and super metabolic regulator, however, whether AMPK has an effect on collagen production in CVB3-infected heart remains to be elucidated. In the present study, the association between AMPK activation and CVB3-infected neonatal rat cardiac fibroblasts (NRCFs) was investigated. Collagen production was determined by the hydroxyproline content of the supernatant and by the expression of type I/IV collagen in the cell lysate. Rat hydroxyproline ELISA was used to detect hydroxyproline content in the supernatant. The expression of type I/IV collagen, and the phosphorylation of AMPK $\alpha-$ Thr $^{172}$ and p38 in the cell lysate were evaluated using western blotting. As expected, it was found that the hydroxyproline content in the supernatant, and the production of collagen I/IV in the cell lysate were significantly promoted at $48 \mathrm{~h}$ post-CVB3-infection. However, this effect was inhibited in a dose-dependent manner when pretreated with 5-aminoimidazole-4-carboxamide-1-4-ribofuranoside (AICAR) for $2 \mathrm{~h}$ prior to CVB3-infection. However, if the cells were preincubated with compound C or SB203580 for 30 min prior the treatment with AICAR, the inhibitive effects of AICAR were reversed. The results of the western blotting
\end{abstract}

Correspondence to: Dr Shengyang Jiang, Department of Cardiology, The Third Affiliated Hospital of Nantong University, Wuxi Institute of Integrated Traditional Chinese and Western Medicine, 585 Xing-Yuan North Road, Beitang, Wuxi, Jiangsu 214041, P.R. China

E-mail: jsystar@126.com

Key words: adenosine monophosphate-activated protein kinase, p38, coxsackievirus, cardiac fibroblast, collagen indicated that the phosphorylation of AMPK $\alpha-\mathrm{Thr}^{172}$ and p38 were significantly increased by AICAR in the NRCFs. However, only the phosphorylation of p38 mitogen-activated protein kinase (MAPK) was inhibited by SB203580. In conclusion, AMPK activation reduced collagen production via the p38 MAPK-dependent pathway in the cardiac fibroblasts induced by CVB3. The results of the present study may contribute to identifying an effective therapy for CVB3-induced myocarditis and CVB3-associated dilated cardiomyopathy.

\section{Introduction}

Viral myocarditis is a potentially life-threatening disease. It often results in heart failure and mortality due to cardiac contractile dysfunction, characterized by systolic and diastolic dysfunction $(1,2)$. As a member of the positive-stranded RNA virus family, Picornaviridae, coxsackie virus B3 (CVB3) has been confirmed to be one of the major viruses identified to cause acute viral myocarditis (3). Cardiac fibroblasts, comprising up to $65-70 \%$ of the cells in the myocardium, are important in the pathology of CVB3-induced myocarditis $(4,5)$. It has been reported that CVB3 infection can cause interstitial collagen deposition resulting in cardiac fibrosis (6). The inhibition of cardiac fibroblast formation and collagen synthesis can provide a means of attenuating and preventing cardiac fibrosis and its sequelae (7).

Adenosine monophosphate-activated protein kinase (AMPK) has been considered as a cellular fuel gauge and super metabolic regulator (8). Its protective role during myocardial ischemia has been reported in detail (9), however, whether AMPK has an effect on the inflammatory cardiomyopathy and myocardial fibrosis induced by CVB3 remains to be elucidated. A previous study reported an association between AMPK and CVB3 replication; activation of AMPK was shown to restrict CVB3 replication by inhibiting lipid accumulation (10). Therefore, the present study aimed to investigate the role of AMPK activation in collagen production in CVB3-infected cardiac fibroblasts, as well as the underlying mechanism. 


\section{Materials and methods}

Reagents. 5-aminoimidazole-4-carboxamide-ribonucleoside (AICAR) was purchased from Toronto Research Chemicals (Toronto, ON, Canada). Compound $\mathrm{C}$ was purchased from EMD Biosciences (San Diego, CA, USA), and SB203580 was purchased from Sigma-Aldrich (St. Louis, MO, USA). Rabbit anti-phosphorylated (p)-AMPK $\alpha-\mathrm{Th}^{172}$ (1:1,000; cat. no. 2531), anti-AMPK $\alpha$ (1:1,000; cat. no. 2532), anti-p-p38 (1:1,000; cat. no. 9211) and anti-p38 (1:1,000; cat. no. 9212) polyclonal antibodies were obtained from Cell Signaling Technology, Inc. (Beverly, MA, USA). Mouse anti-collagen I monoclonal antibody (1:200; cat. no. sc-59772), goat anti-collagen IV polyclonal antibody (1:200; cat. no. sc-9301), rabbit anti-glyceraldehyde 3-phosphate dehydrogenase (GAPDH) monoclonal antibody (1:3,000; cat. no. sc-32233) and horseradish peroxidase (HRP)-conjugated goat anti-rabbit (1:6,000; cat. no. sc-2004) and goat anti-mouse (1:6,000; cat. no. sc-2005) secondary antibodies were obtained from Santa Cruz Biotechnology, Inc. (Santa Cruz, CA, USA). Fetal bovine serum (FBS) was obtained from GE Healthcare Life Sciences (Logan, UT, USA).

Determination of viral titers. HeLa cells [CCL-2; American Type Culture Collection (ATCC), Manassas, VA, USA] were used to propagate the CVB3 Nancy strain (ATCC). Firstly, HeLa cells grown to $80 \%$ confluency were washed two times in $10 \mathrm{ml} 1 \mathrm{X}$ phosphate-buffered saline (PBS), then CVB3 solution was added to HeLa cells and incubated at $37^{\circ} \mathrm{C}$ in $5 \%$ $\mathrm{CO}_{2}$ for $1 \mathrm{~h}$. Dulbecco's modified Eagle's medium (DMEM; GE Healthcare Life Sciences) supplemented with $10 \%$ FBS was added and the plate was incubated at $37^{\circ} \mathrm{C}$ in $5 \% \mathrm{CO}_{2}$ for an additional $48 \mathrm{~h}$. Subsequently, the infected HeLa cells were lysed by three cycles of freezing $\left(-80^{\circ} \mathrm{C}\right)$ and thawing $\left(37^{\circ} \mathrm{C}\right)$. Following centrifugation at $3,000 \mathrm{x} \mathrm{g}$ for $10 \mathrm{~min}$ at room temperature, the debris was removed and serial dilutions (10-fold; $\left.1 \times 10^{-1}-10^{-9}\right)$ of the supernatants were prepared using infection medium containing minimal essential medium (GE Healthcare Life Sciences), 2\% FBS, $30 \mathrm{mM} \mathrm{MgCl}$, $100 \mathrm{U} / \mathrm{ml}$ penicillin, $100 \mu \mathrm{g} / \mathrm{ml}$ streptomycin sulfate and $2 \mathrm{mM}$ glutamine. Subsequently, the serial supernatant dilutions were transferred onto subconfluent monolayers of HeLa cells grown in 96-well culture plates containing $100 \mu \mathrm{l}$ infection medium. Following incubation at $37^{\circ} \mathrm{C}$ for $24 \mathrm{~h}$, the cells were stained with $0.5 \%$ crystal violet (in water) for $5 \mathrm{~min}$ and visualized using an inverted microscope (Olympus IX73; Olympus Corporation, Tokyo, Japan). The 50\% tissue culture infectious dose (TCID50) was calculated using the Reed-Muench method (11).

Cell isolation and culture. The cultured neonatal rat cardiac fibroblasts (NRCFs) were isolated from 1-day-old Sprague-Dawley rats. Briefly, following anesthesia with 2\% ether, the chest cavities of the neonatal rats were opened and the rat hearts were harvested. Subsequently, the neonatal rat hearts were minced and digested using $0.1 \%$ trypsin (Roche Diagnostics GmbH, Mannheim, Germany) and $0.03 \%$ collagenase II (Worthington Biochemical Corporation, Lakewood, NJ, USA). The collected cells were seeded into $10-\mathrm{cm}$ cell culture plates containing $10 \mathrm{ml}$ DMEM supplemented with $1 \%$ penicillin-streptomycin and $10 \% \mathrm{FBS}$. In order to allow the fibroblasts to attach to the cell culture plates, the cell suspension was placed in a $37^{\circ} \mathrm{C} 5 \% \mathrm{CO}_{2}$ incubator for $60 \mathrm{~min}$. Subsequently, the fibroblasts were washed twice with DMEM and cultured in DMEM with 10\% FBS at $37^{\circ} \mathrm{C}$ for $48 \mathrm{~h}$ until they reached confluence. All the NRCFs were incubated in DMEM with 2\% FBS for $24 \mathrm{~h}$ prior to performing the respective experiments. The animal care and experimental protocols were in compliance with the Animal Management Rule of the People's Republic of China (The State Council of the people's Republic of China; http://www. gov.cn/gongbao/content/2014/content_2692743.htm). The present study was approved by the Committee on Ethics of Biomedicine Research at the Third Affiliated Hospital of Nantong University (Wuxi, China).

Hydroxyproline measurement. The cell culture supernatants were collected following treatment, following which the levels of hydroxproline were detected using a hydroxyproline ELISA kit (cat. no. KB2847A; Kaibo Biochemical Reagents Co., Ltd., Shanghai, China), according to the manufacturer's protocol. Hydroxyproline concentrations were determined by the optical density values at $450 \mathrm{~nm}$ on an ELISA plate reader (Bio-Rad Laboratories, Inc., Hercules, CA, USA).

Western blot analysis. Following washing once with cold PBS, the cell samples were lysed in buffer containing $10 \mathrm{mmol} / \mathrm{l}$ sodium pyrophosphate, $100 \mathrm{mmol} / 1 \mathrm{NaCl}, 50 \mathrm{mmol} / \mathrm{l} \mathrm{NaF}$, $1 \mathrm{mmol} / \mathrm{l}$ sodium vanadate, $5 \mathrm{mmol} / 1 \mathrm{EDTA}, 1 \%$ sodium deoxycholate, $20 \mathrm{mmol} / 1$ Tris- $\mathrm{HCl}$ ( $\mathrm{pH} 7.4), 0.1 \%$ sodium dodecyl sulphate (SDS), $1 \mathrm{mmol} / 1$ phenylmethylsulphonyl fluoride, $0.1 \mathrm{mmol} / 1$ aprotinin, $1 \mathrm{mmol} / 1$ leupeptin, $1 \%$ Triton $\mathrm{X}-100$ and $10 \%$ glycerol. The protein concentrations were estimated using a bicinchoninic acid protein assay kit (Pierce Biotechnology, Inc., Rockford, IL, USA). Equal quantities of the protein samples $(30 \mu \mathrm{g})$ were mixed with an equal volume of $2 \mathrm{X}$ SDS sample buffer containing $4 \%$ SDS, $100 \mathrm{mM}$ Tris- $\mathrm{HCl}$ (pH 6.8), 20\% glycerol, $200 \mathrm{mM}$ DTT and $0.2 \%$ bromophenol blue. After boiling the mixture at $95^{\circ} \mathrm{C}$ for $5 \mathrm{~min}$, the protein samples were loaded and separated on 8 or $10 \%$ SDS-polyacrylamide gels. Subsequently, the samples were electroblotted onto nitrocellulose membranes (Pall Life Sciences, East Hill, NY, USA). Nonspecific binding was then blocked with $5 \%$ nonfat milk. The membranes were incubated overnight at $4^{\circ} \mathrm{C}$ with rabbit anti-p-AMPK $\alpha$-Thr ${ }^{172}$ $(1: 1,000)$, anti-AMPK $\alpha(1: 1,000)$, anti-p-p38 $(1: 1,000)$ and anti-p38 $(1: 1,000)$ polyclonal antibodies, mouse anti-collagen I monoclonal antibody (1:200), goat anti-collagen IV polyclonal antibody (1:200) and rabbit anti-GAPDH monoclonal antibody $(1: 3,000)$, followed by incubation with HRP-conjugated goat anti-rabbit and anti-mouse secondary antibodies $(1: 6,000)$ for $1 \mathrm{~h}$ at room temperature. An enhanced chemiluminescence system (ECL kit; Pierce Biotechnology, Inc.) was used to visualize the membranes. The following primary antibodies were used, at 1:1,000 dilutions: Anti-collagen I, anti-collagen IV, anti-AMPK $\alpha$, anti-p-AMPK $\alpha\left(\right.$ Thr $\left.^{172}\right)$, anti-p38, anti-p-p38 and anti-GAPDH.

Statistical analysis. All the experiments were performed at least three times. Data are expressed as the mean \pm stan- 
A

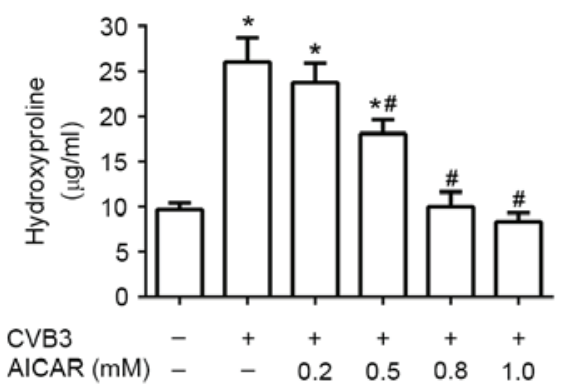

B
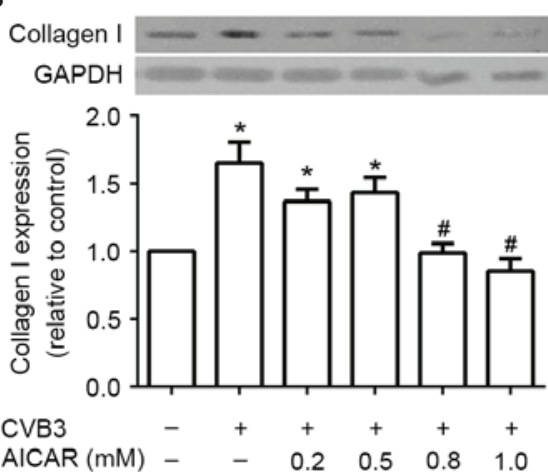

C

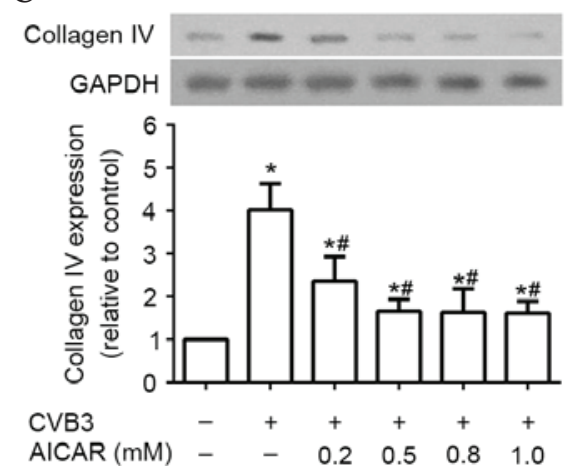

Figure 1. AICAR reduces collagen production in NRCFs induced by CVB3. The cells were infected by CVB3 for $48 \mathrm{~h}$ following treatment with AICAR $(1 \mathrm{mM})$ for $2 \mathrm{~h}$, following which (A) hydroxyproline content in the supernatant, and the expression levels of (B) collagen I and collagen (C) collagen IV in the cell lysate were detected. GAPDH served as an internal control for normalization. Data are presented as the mean \pm standard error of the mean ( $=3$ ). ${ }^{*} \mathrm{P}<0.05$ vs. the control group; ${ }^{\text {}} \mathrm{P}<0.05$ vs. the CVB3 group. CVB3, coxsackie virus B3; NRCFs, neonatal rat cardiac fibroblasts; AICAR, 5-aminoimidazole-4-carboxamide-1-4-ribofuranoside; GAPDH, glyceraldehyde 3-phosphate dehydrogenase.

A

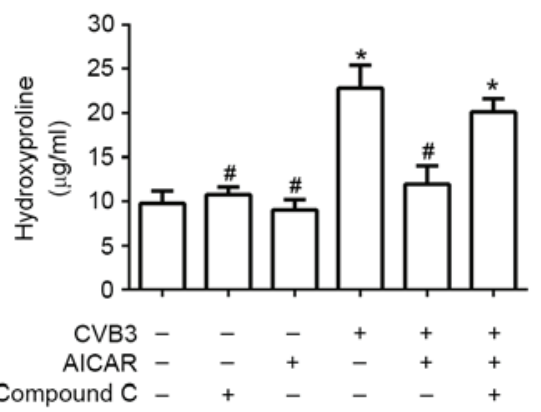

B
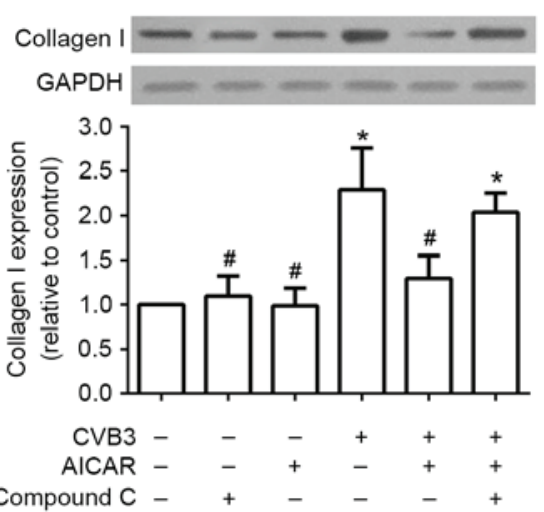

C

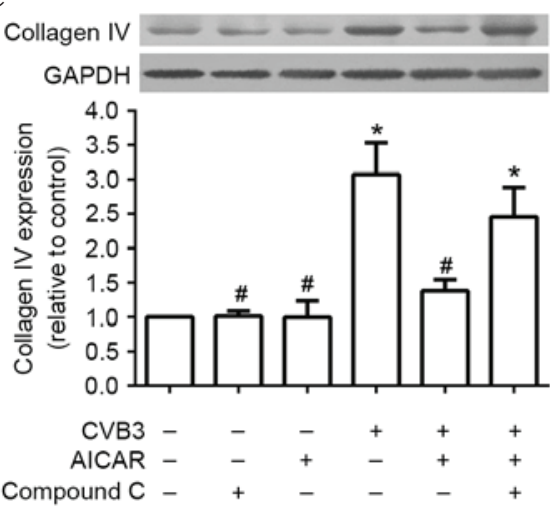

Figure 2. Effects of AICAR are inhibited by compound C in NRCFs infected by CVB3. The NRCFs were preincubated with compound C (1 $\mu \mathrm{M})$ for $30 \mathrm{~min}$, followed by treatment with AICAR (1 mM) and infection with CVB3 for $48 \mathrm{~h}$. (A) Hydroxyproline content in the supernatant, and the expression levels of (B) collagen I and (C) collagen IV in the cell lysate were detected. GAPDH served as an internal control for normalization. Data are presented as the mean \pm standard error of the mean $(n=3)$. ${ }^{*} \mathrm{P}<0.05$ vs. the control group; $\mathrm{P}<0.05$ vs. the $\mathrm{CVB} 3$ group. $\mathrm{CVB} 3$, coxsackie virus $\mathrm{B} 3$; NRCFs, neonatal rat cardiac fibroblasts; AICAR, 5-aminoimidazole-4-carboxamide-1-4-ribofuranoside; GAPDH, glyceraldehyde 3-phosphate dehydrogenase.

dard error of the mean. One-way analysis of variance was performed for multiple-group comparisons, and unpaired two-tailed $t$-tests were used to evaluate the significance between groups. Statistical analyses were performed using SPSS software 20.0 (IBM SPSS, Armonk, NY, USA). P<0.05 were considered to indicate a statistically significant difference.

\section{Results}

AICAR reduces collagen production in CVB3-induced NRCFs in a dose-dependent manner. Although a substantial volume of data regarding AMPK and collagen secretion is now available, their association in NRCFs induced by CVB3 remains to be fully elucidated. In the present study, it was hypothesized that the activation of AMPK may affect the production of collagen in the NRCFs infected with CVB3. Following treatment with AICAR for $30 \mathrm{~min}, 5 \times 10^{4}$ NRCFs incubated in DMEM with $2 \%$ FBS were infected with CVB3 (100 TCID50) for $48 \mathrm{~h}$. As expected, CVB3 infection promoted the levels of hydroxyproline in the supernatant (Fig. 1A) and the production of collagen I/collagen
IV (Fig. 1B and C) in the cell lysate, compared with the control group. However, these effects were dose-dependently inhibited following pretreatment with AICAR.

Inhibitory effects of AICAR are reversed by compound $C$. To examine whether AMPK is a involved in the inhibitory effects of AICAR, the cardiac fibroblasts of mice $\left(5 \times 10^{4}\right.$ per well) were preincubated with $1 \mu \mathrm{M}$ compound $\mathrm{C}$, an AMPK inhibitor, for 30 min, followed by treatment of the cells with AICAR (1 mM) and CVB3 (100 TICD50), as above. In the NRCFs preincubated with compound $\mathrm{C}$, return of the CVB3-induced increases in hydroxyproline content in the supernatant (Fig. 2A) and the production of collagen I/collagen IV (Fig. 2B and C) in the cell lysate were observed.

P38 mitogen-activated protein kinase (MAPK) is a downstream kinase of AMPK in NRCFs. It has been reported that p38 MAPK is an important regulator in collagen production of fibroblasts (12). Therefore, the present study examined whether $\mathrm{p} 38$ MAPK was involved in the inhibitory effects of AICAR in the CVB3-infected NRCFs. It was observed that AMPK and p38 
A

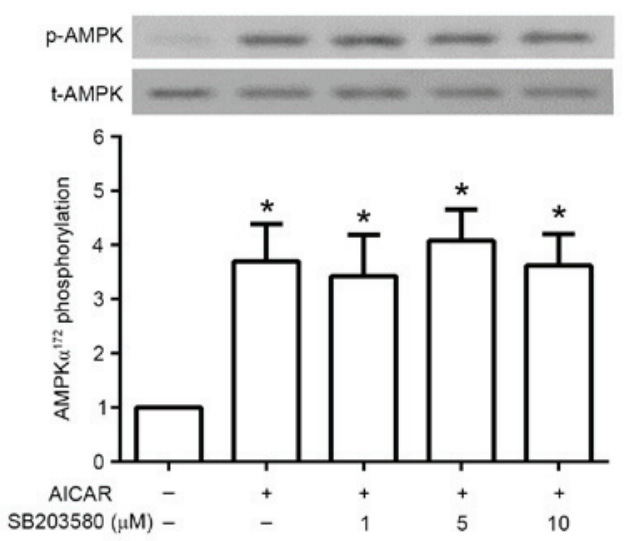

B

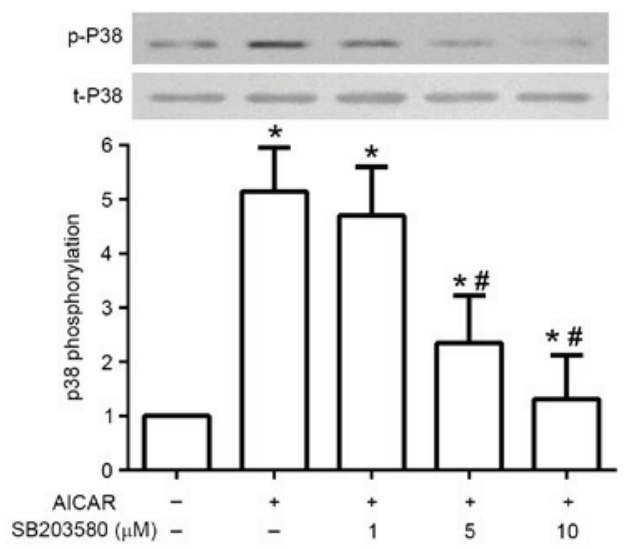

Figure 3. SB203580 inhibits the phosphorylation of p38 mitogen-activated protein kinase in a dose-dependent manner, but not AMPK. Cells pre-incubated with or without SB203580 at various concentrations for $30 \mathrm{~min}$ were treated with AICAR $(1 \mathrm{mM})$ for $48 \mathrm{~h}$. The cell lysates were then immunoblotted with antibodies against (A) p-AMPK $\left(\alpha-\right.$ Thr $\left.^{172}\right)$, AMPK $\alpha,(B)$ p-p38 and total p38. Data are presented as the mean \pm standard error of the mean $(\mathrm{n}=3) .{ }^{*} \mathrm{P}<0.05$ vs. the control group; ${ }^{\#} \mathrm{P}<0.05$ vs. the AICAR group. AICAR, 5-aminoimidazole-4-carboxamide-1-4-ribofuranoside; AMPK. adenosine monophosphate-activated protein kinase; $\mathrm{p}$-, phosphorylated; t-, total.

phosphorylation were significantly increased by AICAR in the NRCFs (Fig. 3A). SB03580, a p38 MAPK-specific inhibitor, inhibited p38 MAPK phosphorylation in a dose-dependent manner, however, AMPK was not affected (Fig. 3B).

P38 MAPK is required for the inhibitory effect of AICAR on collagen production of NRCFs induced by CVB3. To confirm the hypothesis that p38 MAPK is required for the inhibitory effects of AICAR in the collagen production of NRCFs induced by CVB3, the NRCFs were pre-incubated with SB203580 $(10 \mu \mathrm{M})$ for $30 \mathrm{~min}$. The cells were then treated with AICAR (1 mM) and CVB3 (100 TICD50) in turn, as described above. Similar effects to those of compound $\mathrm{C}$ were observed. The CVB3-induced increases in hydroxyproline content in the supernatant (Fig. 4A) and production of collagen I/collagen IV (Fig. 4B and C) in the cell lysate returned following pretreatment with SB203580.

\section{Discussion}

CVB3 infection commonly induces viral myocarditis and dilated cardiomyopathy, eventually resulting in heart failure (13).
$\mathbf{A}$

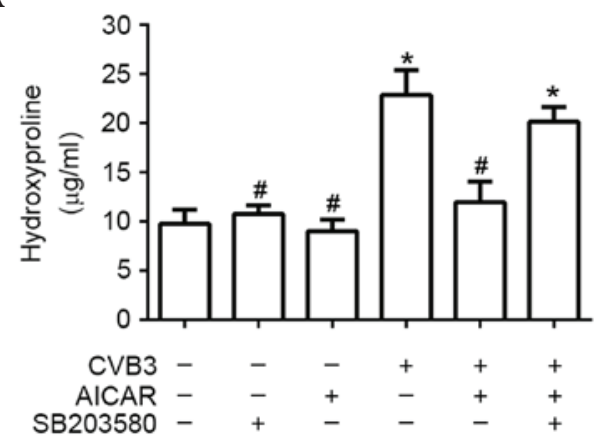

B

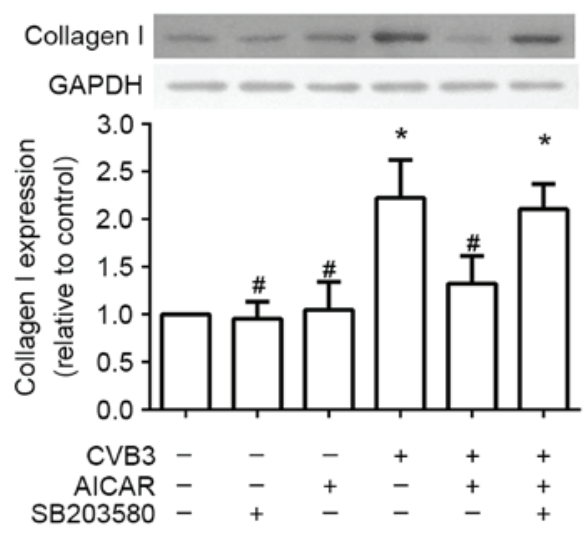

C

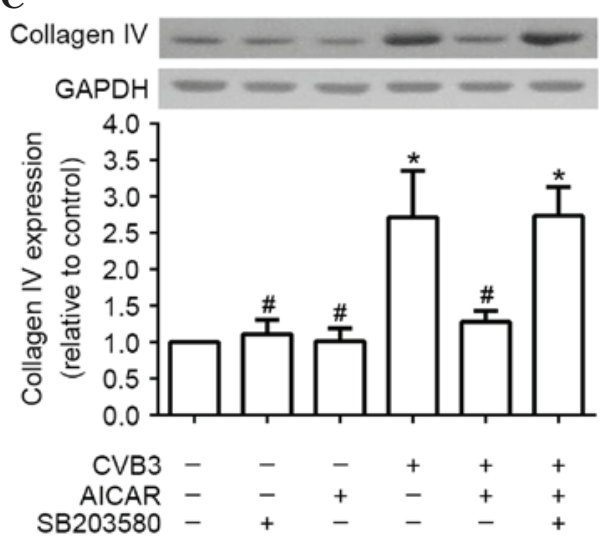

Figure 4. SB203580 reverses the effects of AICAR in NRCFs infected with CVB3. The NRCFs were treated with AICAR $(1 \mathrm{mM})$ following pre-incubation with SB203580 $(10 \mu \mathrm{M})$ for $30 \mathrm{~min}$, after which the cells were treated with AICAR and infected with CVB3. (A) Hydroxyproline content in the supernatant, and the expression levels of (B) collagen I and (C) collagen IV in the cell lysate were detected. GAPDH served as an internal control for normalization. Data are presented as the mean \pm standard error of the mean $(\mathrm{n}=3)$. ${ }^{*} \mathrm{P}<0.05$ vs. the control group; ${ }^{\#} \mathrm{P}<0.05$ vs. the CVB3 group. AICAR, 5-aminoimidazole-4-carboxamide-1-4-ribofuranoside; AMPK, adenosine monophosphate-activated protein kinase; CVB3, coxsackie virus B3; NRCFs, neonatal rat cardiac fibroblasts; GAPDH, glyceraldehyde 3-phosphate dehydrogenase.

Interstitial collagen deposition and the fibrotic remodelling of cardiac tissues are key determinants in the progression of heart failure (14). Cardiac fibroblasts, which produce and degrade the myocardial extracellular matrix, are critical in cardiac inflammation and maladaptive ventricular remodeling. Cardiac fibroblasts 
are involved in the pathology of CVB3-induced myocarditis and dilated cardiomyopathy by promoting cell-specific viral replication (5), aggravating collagen deposition (15) and immunolesions (16). Hydroxyproline, a major component of collagen, can stabilize the triple-helical structure of collagen via maximizing interchain hydrogen bond formation (17). The measurement of hydroxyproline levels has been used as an indicator of collagen content (18). In the present study, it was observed that CVB3 infection promoted hydroxyproline content in the supernatant and the production of collagen I/collagen IV in the cell lysate, compared with the control group.

AMPK, a heterotrimeric complex with a catalytic $(\alpha)$ and two regulatory subunits ( $\beta$ and $\gamma$ ) has been confirmed as a key regulator of cellular energy homeostasis (19). It monitors the AMP/ATP ratio to regulate cellular metabolism by restoring levels of ATP. The $\alpha$-subunit, containing a serine-threonine kinase domain, is essential for AMPK activity. It has a critical activating residue within the catalytic cleft $\left(\mathrm{Thr}^{172}\right)(20,21)$. The phosphorylation status of $\alpha-\mathrm{Thr}^{172}$ has been used as an indicator of the state of AMPK activation (22). AMPK can be activated by a variety of signals, which are not directly associated with metabolism, including ischemia (22), hypoxia, oxidative stress, catecholamines and nitric oxide (23). Furthermore, a variety of signal transduction proteins, including endothelial nitric oxide synthase (24), c-Jun N-terminal kinase (25) and p38MAPK (26), can be activated by AMPK. It has been reported that AMPK is involved in certain heart diseases, including myocardial ischemia $(22,27)$, pressure overload or thyroid hormone-induced cardiac hypertrophy $(28,29)$ and heart failure $(30)$. However, whether AMPK activation is involved in viral myocarditis induced by CVB3 remains to be elucidated.

In the present study, the production of collagen in NRCFs induced by CVB3 was dose-dependently inhibited by AICAR, a well-known pharmacological activator of AMPK. Pretreatment with compound C, an AMPK inhibitor, and SB203580 a p38 inhibitor, reversed the effects of AICAR. AICAR significantly increased the levels of AMPK $\alpha-\mathrm{Thr}^{172}$ and p38 MAPK phosphorylation. However, only p38 MAPK phosphorylation was inhibited by SB203580. These results indicated that $\mathrm{p} 38$ may be the downstream kinase of AMPK in CVB3-infected NRCFs. The pharmacological activation of AMPK reduced collagen production via the p38 MAPK-dependent pathway in the cardiac fibroblasts induced by CVB3. The secretion of collagen by cardiac fibroblasts is a key cause of myocardial fibrosis (31). This finding suggests that AMPK, in addition to its effects on glucose metabolism, may be involved in the cardiac fibrosis observed in the CVB3-infected heart.

In conclusion, the present study demonstrated that the activation of AMPK with AICAR inhibited collagen production via the p38 MAPK-dependent pathway in CVB3-infected NRCFs. The inhibitory effects of AMPK activation in the production of collagen in CVB3-infected cardiac fibroblasts may contribute to identifying an effective therapy for CVB3-induced myocarditis and CVB3-associated dilated cardiomyopathy.

\section{Acknowledgements}

This study was supported by the Natural Science Foundation of China (grant no. 81200161) and the Wuxi Hospital Management Center Project (grant no. YGZXM14012).

\section{References}

1. Ellis CR and Di Salvo T: Myocarditis: Basic and clinical aspects. Cardiol Rev 15: 170-177, 2007.

2. Magnani JW and Dec GW: Myocarditis: Current trends in diagnosis and treatment. Circulation 113: 876-890, 2006.

3. Sagar S, Liu PP and Cooper LT Jr: Myocarditis. Lancet 379: 738-747, 2012.

4. Eghbali M: Cardiac fibroblasts: Function, regulation of gene expression and phenotypic modulation. Basic Res Cardiol 87 (Suppl 2): S183-S189, 1992

5. Lindner D, Li J, Savvatis K, Klingel K, Blankenberg S, Tschöpe C and Westermann D: Cardiac fibroblasts aggravate viral myocarditis: Cell specific coxsackievirus B3 replication. Mediators Inflamm 2014: 519528, 2014.

6. Cao Y, Xu W and Xiong S: Adoptive transfer of regulatory T cells protects against Coxsackievirus B3-induced cardiac fibrosis. PLoS One 8: e74955, 2013.

7. Swaney JS, Roth DM, Olson ER, Naugle JE, Meszaros JG and Insel PA: Inhibition of cardiac myofibroblast formation and collagen synthesis by activation and overexpression of adenylyl cyclase. Proc Natl Acad Sci USA 102: 437-442, 2005.

8. Zaha VG and Young LH: AMP-activated protein kinase regulation and biological actions in the heart. Circ Res 111: 800-814, 2012.

9. Horman S, Beauloye C, Vanoverschelde JL and Bertrand L: AMP-activated protein kinase in the control of cardiac metabolism and remodeling. Curr Heart Fail Rep 9: 164-173, 2012.

10. Xie W, Wang L, Dai Q, Yu H, He X, Xiong J, Sheng H, Zhang D, Xin R, Qi Y, et al: Activation of AMPK restricts coxsackievirus B3 replication by inhibiting lipid accumulation. J Mol Cell Cardiol 85: 155-167, 2015.

11. Reed LJ and Muench H: A simple method of estimating fifty percent endpoints. Am J Hyg 27: 493-497, 1938.

12. Yu XY, Qiao SB, Guan HS, Liu SW and Meng XM: Effects of visfatin on proliferation and collagen synthesis in rat cardiac fibroblasts. Horm Metab Res 42: 507-513, 2010.

13. Liu PP and Yan AT: Cardiovascular magnetic resonance for the diagnosis of acute myocarditis: Prospects for detecting myocardial inflammation. J Am Coll Cardiol 45: 1823-1825, 2005.

14. Pchejetski D, Foussal C, Alfarano C, Lairez O, Calise D, Guilbeau-Frugier C, Schaak S, Seguelas MH, Wanecq E, Valet $\mathrm{P}$, et al: Apelin prevents cardiac fibroblast activation and collagen production through inhibition of sphingosine kinase 1 . Eur Heart J 33: 2360-2369, 2012.

15. Leipner C, Grün K, Müller A, Buchdunger E, Borsi L, Kosmehl H, Berndt A, Janik T, Uecker A, Kiehntopf M and Böhmer FD: Imatinib mesylate attenuates fibrosis in coxsackievirus b3-induced chronic myocarditis. Cardiovasc Res 79: 118-126, 2008

16. Yu M, Hu J, Zhu MX, Zhao T, Liang W, Wen S, Li HH, Long Q, Wang M, Guo HP, et al: Cardiac fibroblasts recruit Th17 cells infiltration into myocardium by secreting CCL20 in CVB3-induced acute viral myocarditis. Cell Physiol Biochem 32: 1437-1450, 2013.

17. Bhattacharjee A and Bansal M: Collagen structure: The Madras triple helix and the current scenario. IUBMB Life 57: 161-172, 2005.

18. Kasyanov V, Moreno-Rodriguez RA, Kalejs M, Ozolanta I, Stradins P, Wen X, Yao H and Mironov V: Age-related analysis of structural, biochemical and mechanical properties of the porcine mitral heart valve leaflets. Connect Tissue Res 54: 394-402, 2013.

19. Puthanveetil P, Wang F, Kewalramani G, Kim MS, Hosseini-Beheshti E, Ng N, Lau W, Pulinilkunnil T, Allard M, Abrahani A and Rodrigues B: Cardiac glycogen accumulation after dexamethasone is regulated by AMPK. Am J Physiol Heart Circ Physiol 295: H1753-H1762, 2008.

20. Liu WY and Jiang RS: Advances in the research of AMPK and its subunit genes. Pak J Biol Sci 16: 1459-1468, 2013.

21. Xiao B, Sanders MJ, Underwood E, Heath R, Mayer FV, Carmena D, Jing C, Walker PA, Eccleston JF, Haire LF, et al: Structure of mammalian AMPK and its regulation by ADP. Nature 472: 230-233, 2011.

22. Baron SJ, Li J, Russell RRIII, Neumann D, Miller EJ, Tuerk R, Wallimann T, Hurley RL, Witters LA and Young LH: Dual mechanisms regulating AMPK kinase action in the ischemic heart. Circ Res 96: 337-345, 2005. 
23. Novikova DS, Garabadzhiu AV, Melino G, Barlev NA and Tribulovich VG: AMP-activated protein kinase: Structure, function, and role in pathological processes. Biochemistry (Mosc) 80: 127-144, 2015.

24. Kar R, Kellogg DL III and Roman LJ: Oxidative stress induces phosphorylation of neuronal NOS in cardiomyocytes through AMP-activated protein kinase (AMPK). Biochem Biophys Res Commun 459: 393-397, 2015.

25. Kang S, Chemaly ER, Hajjar RJ and Lebeche D: Resistin promotes cardiac hypertrophy via the AMP-activated protein kinase/mammalian target of rapamycin (AMPK/mTOR) and c-Jun N-terminal kinase/insulin receptor substrate 1 (JNK/IRS1) pathways. J Biol Chem 286: 18465-18473, 2011.

26. Du JH, Xu N, Song Y, Xu M, Lu ZZ, Han C and Zhang YY: AICAR stimulates IL-6 production via p38 MAPK in cardiac fibroblasts in adult mice: A possible role for AMPK. Biochem Biophys Res Commun 337: 1139-1144, 2005.
27. Ma Y, Wang J, Gao J, Yang H, Wang Y, Manithody C, Li J and Rezaie AR: Antithrombin up-regulates AMP-activated protein kinase signalling during myocardial ischaemia/reperfusion injury. Thromb Haemost 113: 338-349, 2015.

28. Li Y, Chen C, Yao F, Su Q, Liu D, Xue R, Dai G, Fang R, Zeng J, Chen $\mathrm{Y}$, et al: AMPK inhibits cardiac hypertrophy by promoting autophagy via mTORC1. Arch Biochem Biophys 558: 79-86, 2014.

29. Jiang SY, Xu M, Ma XW, Xiao H and Zhang YY: A distinct AMP-activated protein kinase phosphorylation site characterizes cardiac hypertrophy induced by L-thyroxine and angiotensin II. Clin Exp Pharmacol Physiol 37: 919-925, 2010.

30. Beauloye C, Bertrand L, Horman S and Hue L: AMPK activation, a preventive therapeutic target in the transition from cardiac injury to heart failure. Cardiovasc Res 90: 224-233, 2011.

31. Yamazaki KG, Gonzalez E and Zambon AC: Crosstalk between the renin-angiotensin system and the advance glycation end product axis in the heart: Role of the cardiac fibroblast. J Cardiovasc Transl Res 5: 805-813, 2012 